\title{
The ALS-associated proteins FUS and TDP-43 function together to affect Drosophila locomotion and life span
}

\author{
Ji-Wu Wang, 1,2 Jonathan R. Brent,, 1,2 Andrew Tomlinson, ${ }^{3}$ \\ Neil A. Shneider, ${ }^{1,4}$ and Brian D. McCabe',2,5

\begin{abstract}
${ }^{1}$ Center for Motor Neuron Biology and Disease, ${ }^{2}$ Department of Pathology and Cell Biology, ${ }^{3}$ Department of Genetics,
\end{abstract} \\ ${ }^{4}$ Department of Neurology, and ${ }^{5}$ Department of Neuroscience, Columbia University Medical Center, New York, New York, USA.
}

\begin{abstract}
The fatal adult motor neuron disease amyotrophic lateral sclerosis (ALS) shares some clinical and pathological overlap with frontotemporal dementia (FTD), an early-onset neurodegenerative disorder. The RNA/DNAbinding proteins fused in sarcoma (FUS; also known as TLS) and TAR DNA binding protein-43 (TDP-43) have recently been shown to be genetically and pathologically associated with familial forms of ALS and FTD. It is currently unknown whether perturbation of these proteins results in disease through mechanisms that are independent of normal protein function or via the pathophysiological disruption of molecular processes in which they are both critical. Here, we report that Drosophila mutants in which the homolog of FUS is disrupted exhibit decreased adult viability, diminished locomotor speed, and reduced life span compared with controls. These phenotypes were fully rescued by wild-type human FUS, but not ALS-associated mutant FUS proteins. A mutant of the Drosophila homolog of TDP-43 had similar, but more severe, deficits. Through cross-rescue analysis, we demonstrated that FUS acted together with and downstream of TDP-43 in a common genetic pathway in neurons. Furthermore, we found that these proteins associated with each other in an RNA-dependent complex. Our results establish that FUS and TDP-43 function together in vivo and suggest that molecular pathways requiring the combined activities of both of these proteins may be disrupted in ALS and FTD.
\end{abstract}

\section{Introduction}

Amyotrophic lateral sclerosis (ALS) is a fatal adult neurodegenerative disorder characterized by progressive motor system dysfunction and loss of cortical and spinal motor neurons. Though predominantly a sporadic disease, $5 \%-10 \%$ of ALS cases are linked to heritable mutations in a diverse range of proteins (1). Recently, mutations in 2 related proteins, $43 \mathrm{kDa}$ transactive response TAR DNA-binding protein-43 (TDP-43) and fused in sarcoma (FUS; also known as TLS), have been associated with both familial ALS (fALS) and frontotemporal dementia (FTD), the second most common early-onset dementia, which shares some clinical and pathological overlap with ALS. TDP-43 was initially identified as the primary component of abnormal protein aggregates found in patients with both sporadic ALS and FTD $(2,3)$. Subsequent to its purification from aggregates, mutations in TDP-43 were found in approximately $4 \%$ of fALS cases. TDP-43 is an evolutionarily conserved protein with 2 RNA recognition motifs (RRMs) and a C-terminal glycinerich region where the majority of ALS- and FTD-associated mutations occur. TDP-43 can shuttle between the nucleus and cytoplasm and has been linked to both DNA transcription and RNA splicing in addition to many other cellular processes $(4,5)$. Following the identification of TDP-43, mutations in FUS were also linked to ALS and FTD cases $(6,7)$. Both dominant and recessive inherited mutations of FUS in fALS have been described (6), and mutations of FUS in fALS may be more common than TDP-43 mutations (8). fALS mutant FUS proteins can aberrantly localize to the cytoplasm

Authorship note: Ji-Wu Wang and Jonathan R. Brent contributed equally to this work.

Conflict of interest: The authors have declared that no conflict of interest exists. Citation for this article: J Clin Invest. 2011;121(10):4118-4126. doi:10.1172/JCI57883.
$(9,10)$, and aggregates of mutant FUS have been reported in the degenerating neurons of ALS patients (11). Like TDP-43, FUS also possesses an RRM motif and a glycine-rich region in addition to QGSY enriched and RGG repeat regions. FUS is also thought to shuttle between the nucleus and cytoplasm and has been implicated in DNA repair, transcriptional regulation, and RNA processing $(4,5)$. Therefore both FUS and TDP-43 localize aberrantly in ALS patients and both proteins have also been implicated in similar molecular processes (12-14).

These findings suggest at least 2 models for how ALS-associated mutations in TDP-43 and FUS could cause disease (15). In one model, TDP-43 or FUS fALS mutations promote deviant protein activities that are toxic to neurons by mechanisms independent of the protein's normal function (16). In an opposing model, TDP-43 and FUS cooperate in activities that are critical for the long-term survival of specific neuronal subtypes, and mutations in either protein disrupt these processes. To determine whether any data support the latter hypothesis, we took advantage of the conservation of both proteins in Drosophila to search for evidence in vivo that FUS and TDP-43 normally function together in a shared molecular genetic pathway.

\section{Results}

Mutants of Caz, the Drosophila homolog of FUS, have defective development. Drosophila has a single FUS homolog with 53\% amino acid identity to human FUS (hFUS) (Supplemental Figure 1A; supplemental material available online with this article; doi:10.1172/JCI57883DS1) encoded by the cabeza (caz) gene on the X chromosome, which previously identified as an RRM domain-encoding gene expressed in neurons (17). We generated a transgenic Drosophila Caz construct, Caz genomic, that included $5^{\prime}$ sequence including the predicted 
A
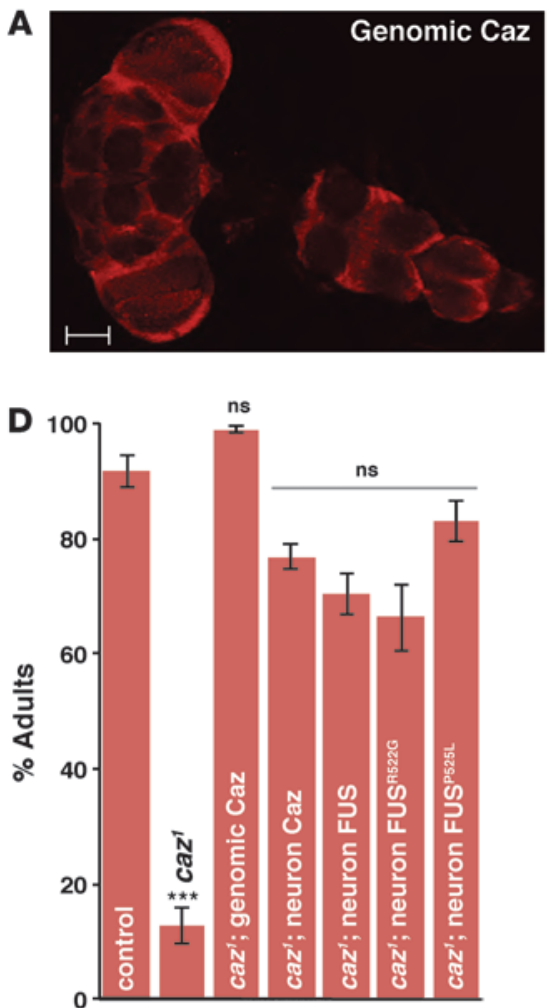

Eclosion

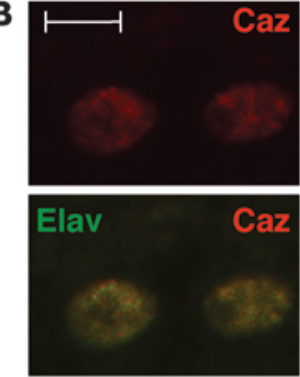

$\mathbf{E}$

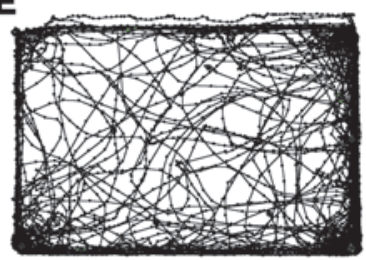

Control

$\mathbf{F}$

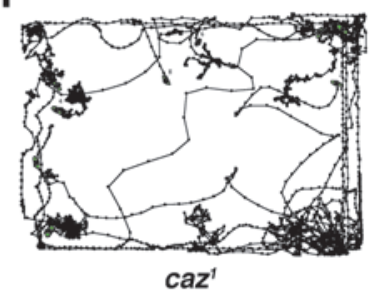

caz' mutants

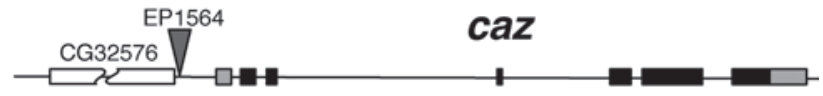

1 . . . . . . . . . . . . . . - $\mathrm{Df}(1) 383$ deletion

CG32576 genomic transgene
G

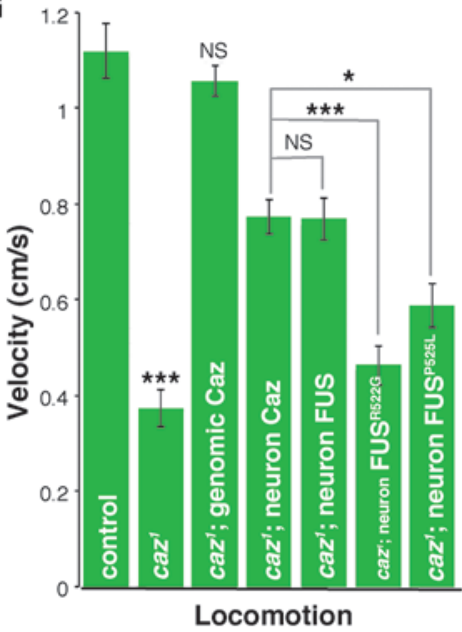

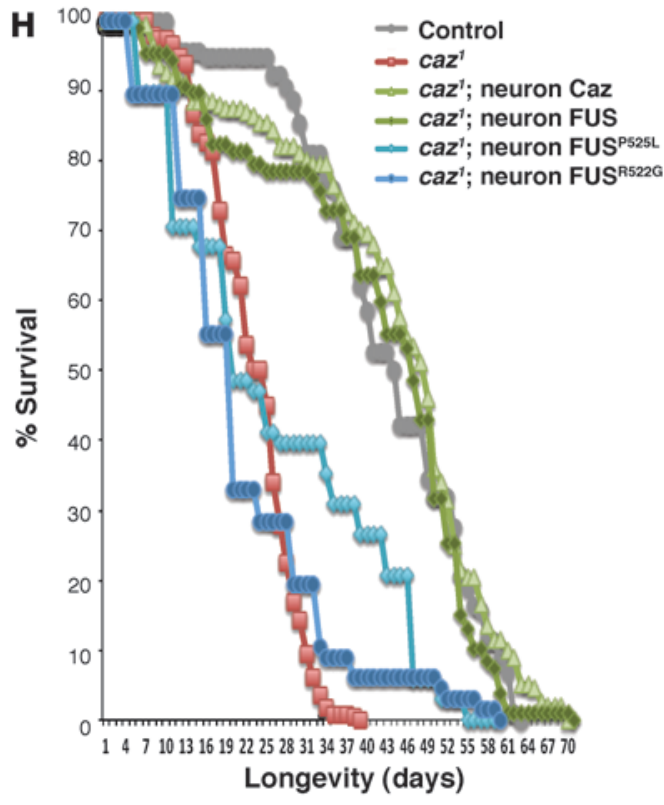

Figure 1

Characterization of Cabeza, the Drosophila homolog of human FUS/TLS. (A) Expression pattern of a caz transgene under the control of the endogenous promoter in the adult brain detected using a FLAG epitope introduced immediately after the start codon. (B) Larval neuronal nuclei expressing genomic Caz detected with FLAG alone (upper panel) or colabeled with the neuronal RNA binding protein Elav. (C) Schematic of caz ${ }^{1}$ mutant construction. The transposon EP1564 was mobilized to create a small deletion $\operatorname{Df}[1] 383$ that removes $58 \%$ of the caz gene, caz promoter sequences, and disrupts the nearby gene CG32576. A rescuing transgene for CG32576 was inserted onto the Df[1]383 chromosome to create caz ${ }^{1}$ mutants. (D) Percentage of male larva of the indicated genotypes that eclosed to produce adults $(n>100)$. Pan-neuronal expression of Caz, human FUS, or ALS mutant FUS (FUS ${ }^{\mathrm{R} 522 \mathrm{G}}$ and FUS ${ }^{\mathrm{P} 25 \mathrm{~L}}$ ) transgenes rescue eclosion equally (genotype: $\mathrm{caz}^{1}$, C155Gal4/Y; UAS transgene). (E) Representative image of 10 superimposed paths of 60 seconds of adult locomotion for control (precise excision) of 1-day-old adult male flies. (F) Representative image of 10 superimposed paths of 60 seconds of adult locomotion of $\mathrm{caz}^{1}$ mutant 1-day-old adult male flies. (G) Walking speed of 1-day-old adult male flies of the indicated genotypes in a 60-second trial $(n>30)$. (H) Percentage survival of adult male flies of the indicated genotypes $(n>68)$. Error bars represent SEM. Scale bars: $100 \mu \mathrm{m}(\mathbf{A}) ; 5 \mu \mathrm{m}$ (B). ${ }^{*} P<0.05 ;{ }^{* \star \star} P<0.001$. caz promoter, full coding region, and $3^{\prime}$ sequences. Into this construct, we additionally inserted a Flag epitope in frame in the cazcoding region. With this transgene, we found that Caz protein was expressed both in neurons (Figure 1A) and nonneuronal cells including glia and muscles (data not shown). Caz protein was localized in the nucleus in both neurons and other cell types (Figure 1B and data not shown) and had a homogenous distribution throughout the nucleus similar to the neuronal RNA-binding protein Elav (Fig- ure $1 \mathrm{~B})$. In order to generate a caz mutant, we mobilized an existing transposable element (EP1564) inserted near caz to generate a small deletion, Df[1] 383, that removed $58 \%$ of the caz gene in addition to 5 ' sequence including the promoter (Figure 1C). This small deletion also disrupted a nearby gene (CG32576), so we generated a rescuing genomic transgene for CG32576 and inserted this on the Df[1]383 chromosome. This combination of Df[1]383 and CG32576 rescuing transgene we named $c a z^{1}$ (Figure 1C). 

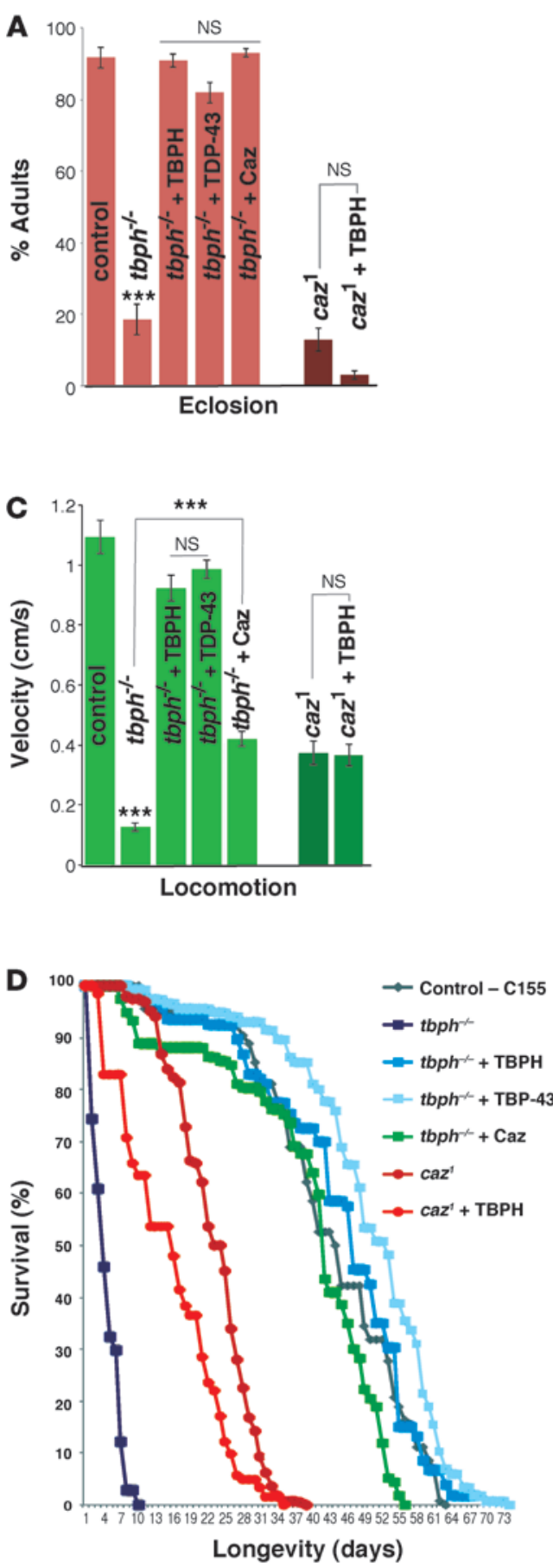

B

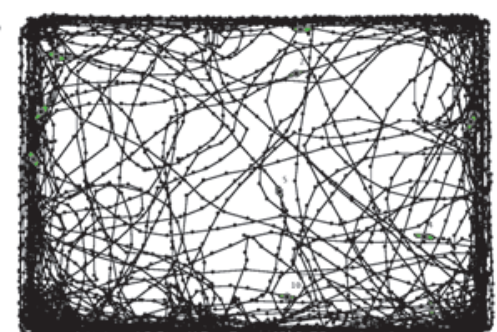

\section{Control}
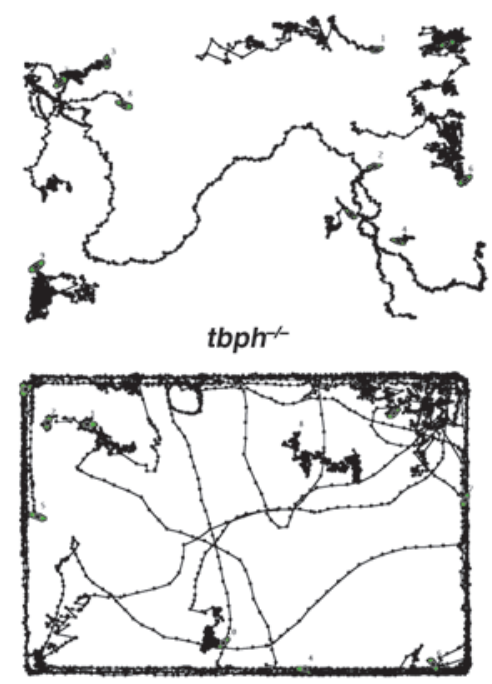

tbph $^{-\alpha}+\mathrm{Caz}$

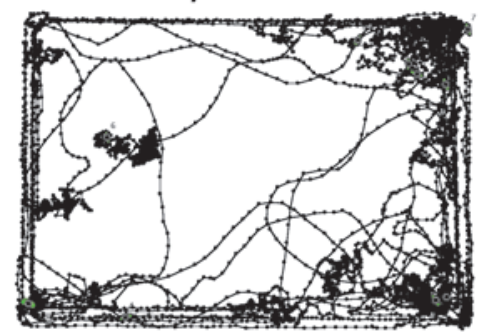

$\mathrm{caz}^{1}$

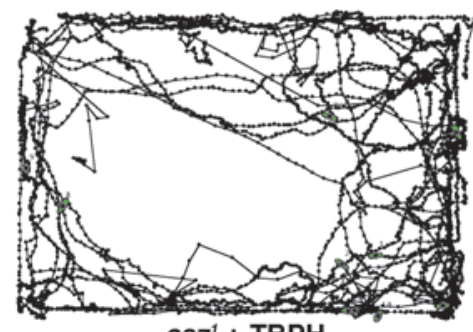

Figure 2

caz and tbph are members of a genetic pathway. (A) Percentage of male larva of the indicated genotypes that eclosed to produce adults $(n>100)$. The tbph $h^{-/}$ genotype is $t b p h^{\Delta 23 / D f[2 R] B S C 660 .}$ + indicates neuronal expression of UASTBPH, UAS-TDP-43, or UAS-Caz with C155-Gal4. (B) Representative images of 10 superimposed paths of 60 seconds of adult locomotion of 1-day-old adult male flies of control, tbph mutant, or caz ${ }^{1}$ mutants either alone or expressing UASCaz or UAS-TBPH. (C) Walking speed of 1-day-old adult male flies of the indicated genotypes in a 60-second trial $(n>30)$. (D) Percentage survival of adult male flies of the indicated genotypes $(n>100)$. Error bars represent SEM. ${ }^{\star \star \star} P<0.001$. $\mathrm{caz}^{1}$ mutant male larva appeared morphologically normal; however, only $14.0 \%(P<0.001)$ successfully completed pupation and eclosed to produce viable adults compared with controls (Figure 1D). This defect was fully rescued by the Caz genomic transgene. We then used the GAL4 system (18) to drive expression of a UAS Caz cDNA transgene in neurons alone. When Caz was restored only in the nervous system by neuronal (C155-GAL4) expression of transgenic UAS Caz, adult viability was also restored to $83.7 \%$ $(P<0.001)$ of control levels, suggesting Caz is predominately but not exclusively required in the nervous system for normal adult eclosion (Figure 1D). We next tested the ability of human FUS to rescue caz mutants by generating a UAS human FUS transgene. Transgenic hFUS was localized in the nucleus of Drosophila motor neurons similar to Caz protein (Supplemental Figure 1B). Expression of hFUS in the nervous system of caz mutants restored adult viability to levels similar to those seen with the expression of Caz, indicating conservation of protein function (Figure 1D). We also generated hFUS ${ }^{\mathrm{R} 522 \mathrm{G}}$ and hFUS ${ }^{\mathrm{P} 525 \mathrm{~L}}$ transgenes based on fALS mutations (6). Using phiC31 transgenesis (19), mutant hFUS transgenes were inserted into the same locus as the wild-type hFUS transgene, and we confirmed they were expressed at identical levels (Supplemental Figure 1C). 

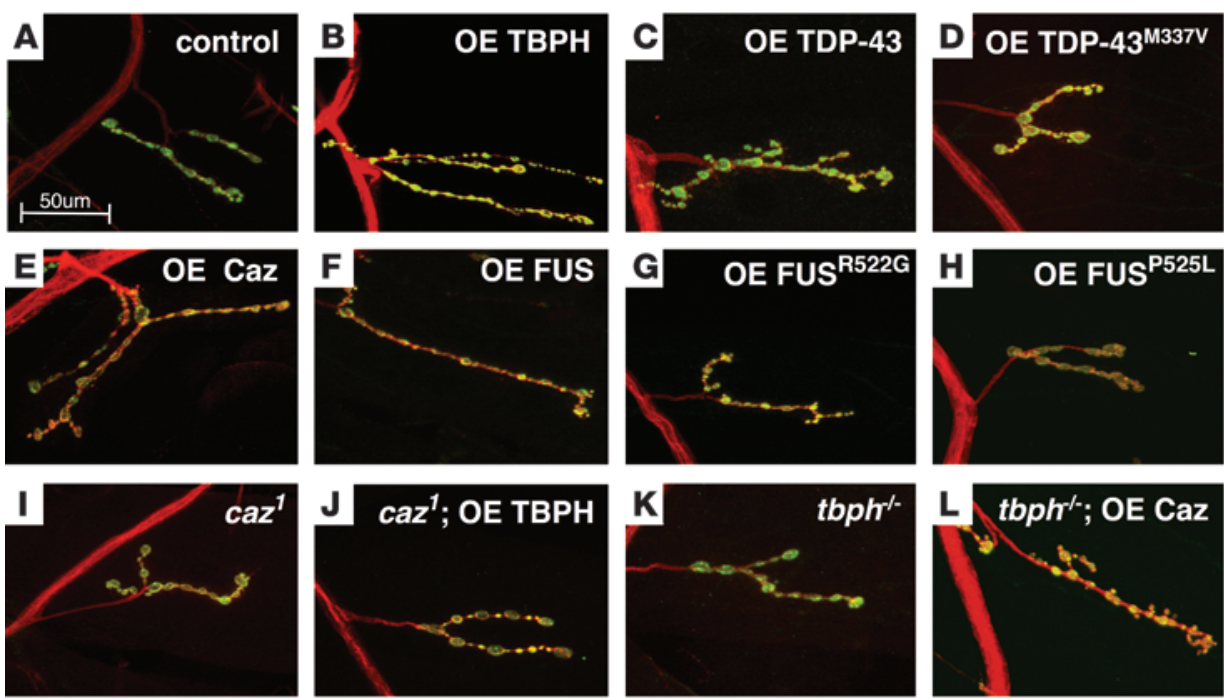

M

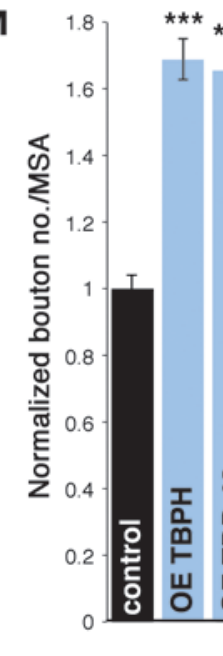

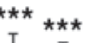

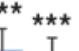

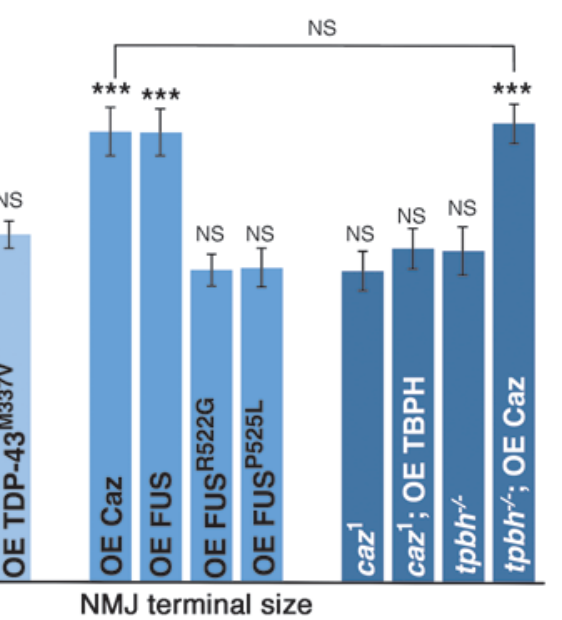

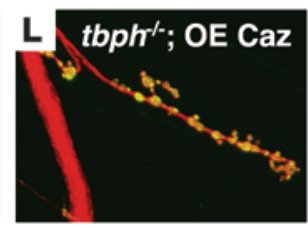

\section{Figure 3}

Caz is epistatic to tbph. (A-L) Third instar NMJ terminals stained with anti-CSP (green) to label the presynapse and anti-HRP (red) to label the neuronal membrane at muscle 4 , segment $\mathrm{A} 3$ for motor neuron overexpressing (OE) transgenic Caz, FUS, TBPH, TDP-43, and FUS or TDP-43 ALS mutants in wild-type $(\mathbf{B}-\mathbf{H})$ or tbph and $\mathrm{caz}^{1}$ mutants ( $\mathbf{J}$ and $\mathbf{L}$ ) driven in motor neurons by OK6-Gal4. The $t b p h^{-1}$ genotype is tbph ${ }^{\Delta 23 / D f[2 R] B S C 660 . ~ O v e r-~}$ expression of wild-type TBPH, TDP43, Caz, or FUS proteins induces NMJ expansion while ALS mutant FUS or TDP-43 ${ }^{\mathrm{M} 337 \mathrm{~V}}$ does not (B-H). Mutants of caz or tbph have normal NMJ morphology (I and K). The NMJ expansion induced by expression of wild-type TDP-43 is completely suppressed in caz mutants $(\mathbf{J})$; however, the NMJ expansion induced by Caz overexpression is not suppressed in tbph mutants (L). (M) Quantification of synapse terminal bouton number divided by muscle surface area for muscle 4 segment $A 3$ normalized to control. Error bars represent SEM. ${ }^{* *} P<0.001$.

Both mutant hFUS proteins also localized to the nucleus of Drosophila motor neurons (Supplemental Figure 1B). Expression of hFUS ${ }^{\mathrm{R} 522 \mathrm{G}}$ or hFUS ${ }^{\mathrm{P} 525 \mathrm{~L}}$ in $c a z$ mutants rescued eclosion to a degree similar to that seen with the expression of Caz, suggesting these mutations do not disrupt the activities of hFUS required to restore Drosophila eclosion (Figure 1D).

caz mutant adults have reduced locomotion and longevity that is not rescued by fALS FUS. We next examined caz ${ }^{1}$ mutant males that survived to adulthood. Externally, these mutants had a mild rough eye defect, abnormal genitalia, and defects in both bristles and wing vein organization, all of which were rescued by the caz genomic transgene (data not shown). Prominently, they also exhibited defects in locomotion. The majority of caz mutants were unable to fly, and compared with controls, they walked slowly, fell over frequently, and had difficulty righting themselves (see Supplemental Videos 1 and 2). We quantified this defect with 2 assays. First, we tested climbing ability using a negative geotaxis assay. In this paradigm, caz mutants had a $55.9 \%$ decrease $(P<0.001)$ in climbing ability compared with controls, which was fully rescued by the Caz genomic transgene (Supplemental Figure 2A). Second, we used quantitative videotracking to measure the locomotor speed of caz mutants and controls (Figure 1, E and G) and observed a 67.6\% rel- ative reduction $(P<0.001)$ in locomotor speed in caz mutants that was also fully rescued by the Caz genomic transgene (Figure 1G). When we restored Caz only in the neurons of caz mutants we could increase locomotor speed by $82 \%(P<0.001)$ over caz mutants alone (Figure $1 \mathrm{G}$ ), but these animals were still significantly slower than controls or animals rescued with genomic Caz, suggesting nonneuronal expression of Caz also contributes to locomotor speed. We next tested to determine whether hFUS could rescue caz mutant locomotion. Expression of wild-type hFUS in caz mutant neurons increased locomotor speed by $75 \%(P<0.001)$ and was not significantly different from rescue with transgenic Caz (Figure $1 \mathrm{G})$. In contrast, however, expression of hFUS ${ }^{\mathrm{R} 522 \mathrm{G}}$ in caz mutant neurons showed no significant improvement of the locomotor speed of caz mutants (Figure 1G). Expression of hFUS ${ }^{\mathrm{P} 525 \mathrm{~L}}$ did increase locomotor speed of caz mutants significantly, but these animals were still $23.6 \%(P<0.05)$ slower than mutants rescued with wild-type hFUS (Figure 1G). Overexpression of wild-type or mutant hFUS in the neurons of control animals had no significant effect on locomotion (Supplemental Figure 2B). These results reveal that, unlike the requirements for FUS during eclosion, fALS mutant isoforms of human FUS are defective in an activity that is essential for normal locomotion in Drosophila. 
A

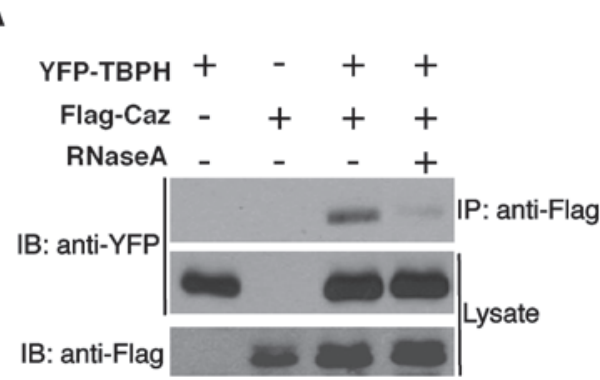

B

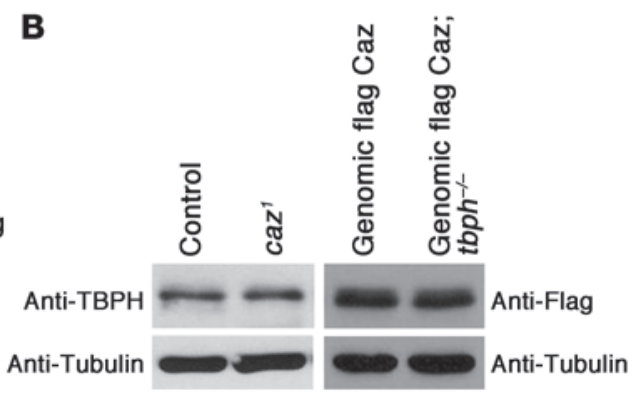

C Human Fus 518 Q D R R E R P Y 526 Drosophila Caz $301 \quad$ G G M R S R P Y 399
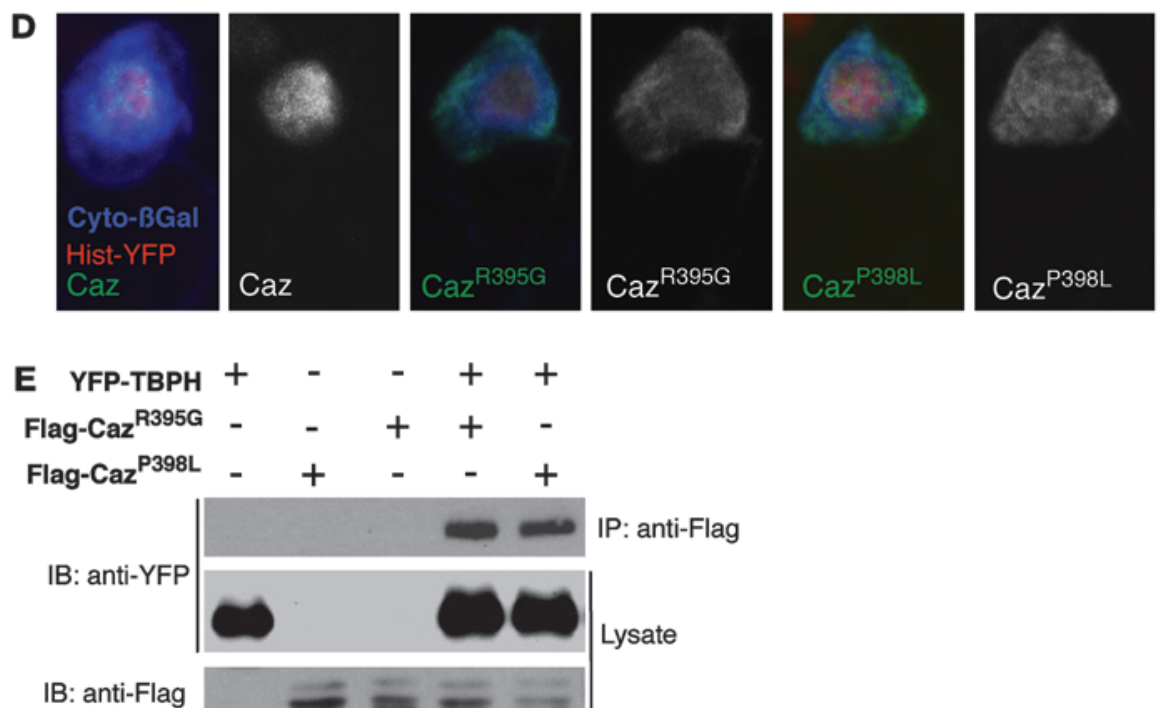

IP: anti-Flag

Lysate

\section{Figure 4}

$\mathrm{Caz}$ and TBPH proteins interact. (A) YFP-tagged TBPH expressed in Drosophila adult brains with $\mathrm{C} 155-$ Gal4 coimmunoprecipitates when flag-tagged Caz is used for pulldown. Treatment of brain extracts with RNaseA inhibits this interaction. (B) Endogenous TBPH protein levels were not changed in 1-day-old caz mutant males compared with precise excision controls, and genomic flagCaz protein is similar in 1-day-old tbph mutants compared with controls. (C) 4 of the 8 C-terminal amino acids of human FUS and Drosophila Caz are identical (green), and 2 (red) were mutated in Caz cDNA transgenes. (D) Drosophila motor neuron cell bodies expressing UAS-Caz, UAS-Caz ${ }^{\mathrm{R} 395 \mathrm{G}}$, and UAS-Caz ${ }^{\mathrm{P} 398 \mathrm{~L}}$ (green), histone-YFP (red), and cytoplasmic $\beta$-galactosidase (blue) driven by the motor neuron driver OK319Gal4 or single channel images of Caz or Caz mutants (gray). Unlike wild-type Caz, both Caz C-terminal mutants are found extensively in the cytoplasm. (E) YFP-TBPH coimmunoprecipitates when flag-tagged $\mathrm{Caz}^{\mathrm{R} 395 \mathrm{G}}$ or $\mathrm{Caz}^{\mathrm{P} 398 \mathrm{~L}}$ is used for pulldown from adult brain extracts.
We further observed that caz mutant males had a shorter life span than control male flies (Figure $1 \mathrm{H}$ ). The average life span of control males was 53 days, whereas caz mutants lived an average of 23 days, a 57\% decrease $(P<0.001)$. The Caz genomic transgene fully restored the average life span of caz mutants to control levels (Supplemental Figure 2C), as did neuronal expression of transgenic Caz or hFUS (Figure 1H). In contrast, expression of hFUSR522G or hFUS ${ }^{\mathrm{P} 525 \mathrm{~L}}$ in caz mutants did not significantly restore median life span, although maximum life span was increased (Figure $1 \mathrm{H}$ ). Overexpression of Caz or hFUS transgenes in control animals had no effect on longevity (Supplemental Figure 2D). Therefore, as was found for locomotion, fALS mutant FUS proteins were deficient in an activity required for longevity in Drosophila. We examined the brain tissue of 25-day-old caz mutants and controls for evidence of neuronal loss (Supplemental Figure 2E) and did not observe vacuolization or other evidence of extensive neuronal death. Interestingly, overexpression of fALS mutant human SOD1 in Drosophila can also inhibit locomotion without inducing neuronal loss (20).

tbph mutants have phenotypes similar to those of caz mutants. The Drosophila homolog of TDP-43, TBPH, is only expressed in neurons $(15,16)$, and protein-null mutants of $t b p h$ can survive to adulthood (ref. 21 and Supplemental Figure 3G). We examined the phenotype of adult transallelic null mutants of tbph to compare them with caz mutants. Unlike in caz mutants, we did not observe defects in eye, wing, or genital development in adult tbph mutants. When we examined the eclosion rate of $t b p h$ mutants, we found that only $19.4 \%(P<0.001)$ of $t p b b$ mutants survived to adulthood compared with controls (Figure $2 \mathrm{~A}$ ). This defect was rescued completely by expression of either transgenic Drosophila TPBH or human TDP43 (hTDP-43) in the neurons of tbph mutants. We also examined adult locomotor speed in adult tbph mutants (Figure 2, B and C). Compared with controls, tbph mutants had a dramatic $88.4 \%$ $(P<0.001)$ reduction of locomotor speed that was also fully rescued by neuronal expression of TBPH or hTDP-43. Finally, we examined the longevity of these mutants (Figure 2D). We found that the marked reduction in the survival of tbph mutants to an average of 4 days $(P<0.001)$ was also restored to control levels by neuronal expression of TBPH or hTDP-43. Therefore, consistent with previous studies (21), we find that tbph mutants, in comparison with caz mutants, have similar, though quantitatively more severe, eclosion, adult locomotion, and longevity phenotypes.

caz and tbph are components of a common genetic pathway in neurons. To determine whether a genetic relationship existed between $c a z$ and $t b p h$, we attempted to cross-rescue $t b p h$ or caz mutants by overexpression of the other gene. First, we expressed transgenic TPBH in the nervous system of caz mutants and 
examined eclosion rate, adult locomotor velocity, and longevity. Expression of TBPH in caz mutants resulted in no alteration in any of these measures when compared with caz mutants alone (Figure 2, A-D). We next overexpressed Caz in tbph mutants. Surprisingly, overexpression of Caz in $t b p h$ mutants restored their eclosion frequency to levels not significantly different from those achieved by expression of TBPH (Figure 2A). Caz overexpression in $t p b b$ mutants also increased adult locomotion velocity by $229.8 \%(P<0.001)$ compared with $t b p h$ mutants alone, although these animals were still significantly slower than mutants rescued with TBPH (Figure 2, B and C). Most dramatically, Caz overexpression had a dramatic effect on tbph mutant longevity, increasing the mean life span of tbph mutants 13 -fold $(P<0.001)$ to levels not significantly different from animals rescued with TBPH expression (Figure 2D). These data indicated that caz and tbph might function in a common genetic pathway, necessary for neuronal development, locomotion, and longevity, in which Caz was epistatic to TBPH.

To test this model further, we searched for gain-of-function phenotypes for Caz and TBPH. Mutants of tbph have been reported to have defects in larval neuromuscular junction (NMJ) synapse morphology (21); however, we were unable to replicate this finding in transallelic combinations of tbph (Figure $3, \mathrm{~K}$ and $\mathrm{M}$ ). In contrast, however, when TBPH or TDP-43 were overexpressed in motor neurons, we did observe a dramatic expansion of NMJ terminal size with a $68.4 \%$ and $65.5 \%(P<0.001)$ increase in the number of synaptic boutons (Figure 3, B, C, and M), respectively. We observed no change when we overexpressed a fALS mutant, TDP-43 ${ }^{\mathrm{M} 337 \mathrm{~V}}$, at identical levels (Figure 3, D and M, and Supplemental Figure $3 \mathrm{H}$ ). Similarly, caz ${ }^{1}$ mutants had normal NMJ morphology (Figure 3, I and M); however, overexpression of wildtype Caz or hFUS did induce an expansion of NMJ size with a $35.3 \%$ or $35.2 \%(P<0.001)$ increase in synaptic bouton numbers, respectively (Figure 3, E, F, and $\mathrm{M}$ ). In contrast, overexpression of hFUS $^{\mathrm{R} 522 \mathrm{G}}$ or hFUS ${ }^{\mathrm{P} 525 \mathrm{~L}}$ did not significantly increase NMJ terminal size (Figure 3, G, H, and M). Thus, NMJ terminal expansion is a common phenotype induced by overexpression of both Caz/hFUS and TBPH/hTDP-43 in motor neurons that is inhibited by fALS mutations. We used this assay to further test the genetic interaction between caz and $t b p h$. We first overexpressed TBPH in the motor neurons of caz mutants. When we did this, the NMJ expansion induced by TBPH overexpression was completely suppressed to control levels (Figure 3, I and M), suggesting that TBPH requires the presence of Caz to induce NMJ terminal expansion. In contrast, when we overexpressed Caz in a tbph mutant, it induced a level of NMJ terminal expansion similar to that observed when Caz was overexpressed in a control background (Figure 3, J and $\mathrm{M})$. This indicates that Caz does not require TBPH to induce NMJ expansion and further supports a model in which Caz functions in a genetic pathway downstream of TBPH.

Caz and TBPH proteins interact in neurons. Human FUS and TDP-43 proteins have been reported to physically interact in mammalian cell culture $(12,14,22)$, so we next determined whether this interaction also occurred in Drosophila neurons. We generated a YFP-tagged TBPH transgene and used it to test for interaction with Flag-tagged Caz in extracts from Drosophila adult brains. We found that when Caz was pulled down, TPBH was coimmunoprecipitated, confirming this protein interaction also occurred in Drosophila neurons (Figure 4A). We next determined whether this interaction was dependent upon RNA by treating brain extracts with RNase prior to coimmunoprecipitation. We found that this dramatically reduced the coimmunoprecipitation of TBPH by Caz, indicating that the interaction of Caz with TBPH is at least partially dependent upon RNA (Figure 4A). We next asked whether $\mathrm{Caz}$ and TBPH are required to regulate the expression or stability of each other. To do this, we examined the protein levels of TBPH in caz mutants and vice versa. We found no difference in the level of TBPH in caz mutant brain extracts using an anti-TBPH antibody (Figure 4B). Similarly, when we introduced the genomic flagtagged Caz transgene into tbph mutants, we also saw no change in Caz protein levels (Figure 4B). Therefore, while Caz and TPBH proteins can interact with each other, neither gene is required to maintain normal levels of the other protein.

The C terminus of FUS has been shown to act as a noncanonical nuclear localization sequence (9). To determine whether homologous amino acids are required for nucleus localization in $\mathrm{Caz}$, we generated UAS Caz transgenes with point mutations that change the conserved C-terminal amino acid arginine 395 to glutamate (Caz $\left.{ }^{\mathrm{R} 395 \mathrm{G}}\right)$ or proline 398 to leucine (Caz ${ }^{\mathrm{P} 398 \mathrm{~L}}$ ) (Figure $\left.4 \mathrm{C}\right)$. These transgenes were inserted in the same locus as wild-type Caz, and expression levels of these mutant transgenes were similar (Supplemental Figure 3I). Like wild-type Caz, overexpression of either mutant protein in neurons did not alter adult eclosion frequency or locomotor velocity (data not shown). However, in contrast to wildtype Caz, both mutant Caz proteins were found to be distributed extensively throughout the cytoplasm in addition to the nucleus when expressed in motor neurons (Figure 4D). Thus, in context of Caz, these C-terminal amino acids are also required to correctly localize the protein only to the nucleus. We then asked whether these mutant Caz proteins could still biochemically interact with TBPH. We found that TBPH could be coimmunoprecipitated by pull-down of either $\mathrm{Caz}^{\mathrm{R} 395 \mathrm{G}}$ and $\mathrm{Caz}^{\mathrm{P} 398 \mathrm{~L}}$, indicating that these proteins retained their ability to interact with TBPH (Figure 4E).

\section{Discussion}

Mutations in FUS and TDP-43 are associated with inherited forms of both ALS and FTD, and similarities in their protein structure and putative functions have led to the suggestion that they could work together in a common molecular or cellular process $(4,5)$. Here, we have generated mutants of caz, the Drosophila homolog of FUS, and shown they have aberrant development, defective adult locomotion, and decreased adult life span. All of these phenotypes can be rescued equivalently by transgenic wild-type Drosophila Caz or human FUS, revealing a remarkable conservation of protein function during evolution. In contrast, FUS transgenes with familial ALS-associated mutations can rescue the survival of caz mutants to adulthood but cannot rescue defects in adult locomotion or longevity. These results indicate that fALS mutations disrupt some of the physiological activities of FUS and reveal a unique vulnerability of adult neurons to fALS mutations.

The mechanisms through which mutations in TDP-43 or FUS cause ALS and FTD are not known, and both toxic gain-of-function and loss-of-function models have been proposed (4, 5). ALSassociated mutant forms of TDP-43 are hyperphosphorylated and aberrantly cleaved to produce fragments that are cytotoxic to cells, and these tend to form abnormal, ubiquitinated cytoplasmic aggregates, in vivo and in vitro $(16,23,24)$. Mutations in FUS can also induce cytosolic aggregates $(6,7,25,26)$, but as with TDP-43, the mechanisms linking FUS mutations to neuronal degeneration are still unknown (15). High-level overexpression of either wild-type 
or mutant TDP-43 is neurotoxic in mice, zebra fish, or Drosophila, which appears to support a toxic gain-of-function model (26-34); however, overexpression of mutant proteins may also perturb the activity of endogenous TDP-43 (35). In contrast, in this study, we have generated transgenic lines in which wild-type Caz, FUS, and FUS mutants are overexpressed at a uniform level in the nervous system to approximately 2-fold over endogenous Caz levels (Supplemental Figure 3D). Inconsistent with toxic gain-of-function of FUS, we did not observe defects in development, locomotion, or longevity from overexpression of either wild-type or mutant proteins at this level. Rather, our evidence supports a model in which fALS mutations result in the loss of at least some activities of the FUS protein. This is supported by 2 lines of evidence: the failure of mutant human FUS to rescue caz mutant defects in locomotion and longevity to a level similar to that of wild-type FUS, and the inability of overexpressed fALS mutant FUS to alter NMJ morphology in contrast with the terminal expansion produced by overexpression of wild-type Drosophila or human proteins. Since mutant human FUS proteins localize correctly to nucleus in Drosophila neurons, our data suggest that fALS mutant FUS has reduced biochemical or cellular activity within the nucleus. Both dominant and recessive FUS mutations have been reported (6), suggesting the possibility that the disruption of the activity of 1 FUS allele could be sufficient to increase the risk for ALS or FTD.

Both FUS and TDP-43 have been linked to multiple molecular processes, many of which involve RNA processing (13). Recently, TDP-43 has been demonstrated to be present in 2 separable protein complexes, a large complex that is enriched in ribosomal proteins and translation factors and a smaller complex that also contains FUS (14). fALS mutants of TDP-43 may also have altered affinity for FUS (12). We find that Drosophila Caz also associates with TBPH in neurons and show that this interaction depends upon RNA. This interaction is not abolished by mutations of Caz that cause the protein to aberrantly localize to the cytoplasm in addition to the nucleus. Beyond this, our cross-rescue analysis reveals that the production of excess Caz has a remarkable ability to rescue the phenotypes of tbph mutants when overproduced in neurons. Overexpression of Caz in tbph mutants completely rescued defects in eclosion and longevity in addition to substantially rescuing adult locomotion. This is consistent with a genetic relationship in which caz is epistatic to or downstream of tbph. This genetic relationship was confirmed by gain-of-function experiments in motor neurons. Our results suggest that the interaction of TBPH with Caz is critical for the activities of TPBH we have examined. Furthermore, some of these activities (locomotion, longevity, NMJ expansion) are also sensitive to fALS-associated FUS mutations. Our data therefore suggest the possibility that in familial forms of ALS and FTD, there is a disruption of molecular processes that require the combined function of both TDP-43 and FUS. If so, defining the nature and components of these processes will be key to understanding the molecular and cellular defects that lead to neural dysfunction in ALS and FTD.

\section{Methods}

Generation of caz $z^{1}$ mutants and rescue strains. To generate a caz mutant, we mobilized the transposon insertion EP1564 (36) near caz to generate a small deletion Df[1] 383 that removed 2443 bp (57.6\%) of the caz gene and $3251 \mathrm{bp}$ of upstream sequence including the entire caz promoter. As this deletion also disrupts the nearby gene CG32576, we generated a rescuing genomic transgene for CG32576 (see below). CG32576 genomic rescue flies were generated by insertion into the attP18 landing site on the $\mathrm{X}$ chromosome. We recombined this transgene to the Df[1]383 chromosome to generate $c a z^{1}$ mutants. For all experiments, hemizygous $c a z^{1} / Y$ males were examined. As a control for all caz experiments, we used males of a sequenceverified precise excision of EP1564. For neuronal rescue of $c a z^{1}$ mutants, C155-Gal4, a pan-neuronal GAL4 enhancer trap near the elav gene (37), was additionally introduced onto the $c a z^{1}$ chromosome by recombination. We used C155-Gal4 males as an additional control in these experiments.

Generation of novel transgenes. All Caz, FUS, TPBH, and TDP-43 transgenes were inserted into the AttP40 site on chromosome III using phiC31 targeted transgenesis (38) (Genetic Services). Identical expression was confirmed by Western blot (Supplemental Figure 3).

Other stocks. The following additional stocks were used: TBPH $\left(t b p b^{\Delta 23}\right)$ (21) (gift from Fabian Feiguin and Francisco Baralle, International Centre for Genetic Engineering and Biotechnology [ICGEB], Trieste, Italy), Df[2R]BSC660 (B\#26512), C155-GAL4 (37), GMR-GAL4 (39), OK6-Gal4 (40), OK319-GAL4, UAS-H1-YFP (41) (gift from Alexei Tulin, Fox Chase Cancer Center, Philadelphia, Pennsylvania, USA), and UAS-LacZ (B\#8530).

Eclosion measurements. Third instar larvae of appropriate genotypes were selected and transferred to fresh vials. Percentage of eclosion was defined as ratio of the number of empty pupal cases to the number of total pupal cases. 90 to 200 pupae were assayed for each genotype.

Locomotion assays. High-resolution locomotor assays were performed using a modified procedure based on Slawson and colleagues' work (42). Briefly, adult male flies were collected between 0 and 12 hours after eclosion and transferred into vials at a density of 10 flies per vial. Animals were never anesthetized, and all experiments were performed at the same time of day to avoid variation due to circadian rhythm. After a 24-hour waiting period, single flies were added to a $40 \mathrm{~mm}$ by $55 \mathrm{~mm}$ acrylic locomotion chamber. After 1 minute, a 60-second clip of the locomotor behavior of the animal was captured using video camera mounted on a dissection microscope connected to a Macintosh computer with Final Cut 6.0. The movies were then converted to QuickTime and were analyzed using Dynamic Image Analysis Software 3.4.2 (Soll Technologies). All analysis was carried out double blind. 10 random traces were combined to produce the data depicted in Figures 1 and 2. Negative geotaxis climbing assays were performed as described in Martinez et al. (43).

Immunohistochemistry. Adult brains and ventral nerve cords were dissected using established techniques (44). Larval brains were dissected and stained as previously described $(45,46)$. They were labeled with mouse anti-flag (1:500) (Sigma-Aldrich) and rat anti-ELAV (1:500) (DSHB). The secondary antibodies used were anti-mouse Cy3 (1:200) (Jackson ImmunoResearch) and anti-rat Cy2 (1:200) (Jackson ImmunoResearch). Larval NMJs of third instar larvae were dissected and stained as previously described $(45,46)$. Terminals were labeled with a mouse anti-CSP primary antibody 6D6 (1:200) (DSHB), anti-mouse Cy3 secondary (1:1000), and an HRP-Cy3 conjugate (1:400) (Jackson ImmunoResearch). Samples were mounted in ProLong Gold Antifade Reagent (Invitrogen). The number of synaptic boutons was counted at segment A3, muscle 4 .

For motor neuron colabeling, the transgenes UAS-H1YFP and UAS-LacZ were driven by OK319-GAL4 together with UAS versions of Flag-CAZ, Flag-FUS, Flag-FUS P525L, Flag-FUSR522G, Flag-CazR395G, or FlagCazP398L. Immunohistochemistry was performed with primary antibodies mouse anti-Flag (1:1000) (Sigma-Aldrich), rabbit anti-BGal (1:1000) (Cappel), and chicken anti-GFP (1:1000) (Abcam) and secondary antibodies anti-mouse $\mathrm{Cy} 3$ (1:1000) anti-rabbit Alexa Fluor 647 (1:1000), and antichicken Alexa Fluor 488 (1:2000).

Molecular biology. To generate FLAG-tagged Caz genomic DNA, a 6.4-kb caz genomic DNA fragment that excludes the CG32576-coding region was cloned into the EcoRI/XbaI sites of $\mathrm{pBI}$, and 3 copies of the FLAG epitope 
sequence (47) were inserted immediately after the Caz start codon by PCR. To generate CG32576 rescue genomic DNA, a 5.0-kb genomic DNA fragment excluding the Caz-coding region was subcloned from BAC CH322-46G07 (BACPAC Resources Center) into the EcoRI/XbaI sites of pBI. Briefly, pBI was constructed by cloning gypsy insulator sequences (48) flanking a multiple cloning site into the pUASTattB vector (49). Untagged UAS transgenes were generated using PBI-UASC-G, which was constructed by introducing 10 copies of the UAS-binding sequence upstream of a Drosophila Synthetic Core Promoter sequence (50) followed by a Gateway recombination cassette (51) and an SV40 polyadenylation sequence into the pBI vector. Tagged UAS transgenes were generated with either PBI-UASC-VG or pBI-UASC-FG, where an $\mathrm{N}$-terminal Venus-YFP sequence (52) or FLAG epitope tag (47), respectively, was introduced in frame with the gateway cassette of PBI-UASC-G. Caz was amplified from cDNA clone LD 27761 (DGRC), TBPH was amplified from cDNA clone GH09868 (DGRC), TDP-43 was amplified from cDNA clone \#30389805 (Open Biosystems), FUS was amplified from cDNA clone \#2822692 (Open Biosystems). Mutations when appropriate were introduced in PCR primers or by site-directed mutagenesis (Stratagene).

Western blot and immunoprecipitation. For Western blots, adult fly heads were homogenized in SDS loading buffer. After protein extracts were cleared by centrifugation at $16,000 \mathrm{~g}$ for 5 minutes, the supernatants were subjected to SDS-PAGE, transferred to Protran Nitrocellulose Membrane (Whatman), hybridized with antibodies, and detected with ECL Western Blotting Substrate (Pierce). Mouse anti-FLAG (M2) (Sigma-Aldrich) was used at 1:1000 dilution, rabbit anti-TBPH at 1:400 (21) (gift from Fabian Feiguin and Francisco Baralle, ICGEB, Trieste, Italy), mouse anti- $\beta$-tubulin (E7) (DSHB) at 1:200, rabbit anti-GFP at 1:2000, and mouse anti-TDP-43
(2E2-D3) (Abnova) at 1:1000. For immunoprecipitation, adult fly heads were homogenized in lysis buffer $(50 \mathrm{mM}$ Tris- $\mathrm{HCl}, \mathrm{pH} 7.4,150 \mathrm{mM} \mathrm{NaCl}$, 2 mM EDTA, 10\% glycerol, 1\% Triton X-100, Protease Inhibitor Cocktail [Roche]) on ice, centrifuged at $16,000 \mathrm{~g}$ for 20 minutes. The supernatants were incubated with anti-FLAG M2-agarose (Sigma-Aldrich) at $4^{\circ} \mathrm{C}$ for 4 hours and then washed with lysis buffer 4 times. Immunocomplexes were denatured in SDS loading buffer and subjected to SDS-PAGE. For RNase treatment, head extracts were incubated with $200 \mu \mathrm{g} / \mathrm{ml}$ RNase A for 30 minutes at $25^{\circ} \mathrm{C}$ before immunoprecipitation.

Statistics. Statistical analysis was carried out using ANOVA with Instat 3.0. $P<0.05$ was considered significant.

Study approval. Drosophila (common fruit fly) studies are not subject to institutional board review.

\section{Acknowledgments}

We are grateful to Fabian Feiguin, Francisco Baralle, and Alexei Tulin for reagents. We thank Serge Przedborski, Chris Henderson, and Erin Savner for comments on the manuscript. A. Tomlinson was funded by NIH EY012536. N.A. Shneider and B.D. McCabe were funded by grants from P2ALS.

Received for publication March 7, 2011, and accepted in revised form July 27, 2011.

Address correspondence to: Brian D. McCabe, CUMC, P\&S 11420, 630 W. 168th St., New York, New York 10032, USA. Phone: 212.305.3548; Fax: 212.305.5775; E-mail: brian@mccabelab.org.
1. Mitchell JD, Borasio GD. Amyotrophic lateral sclerosis. Lancet. 2007;369(9578):2031-2041.

2. Neumann M, et al. Ubiquitinated TDP-43 in frontotemporal lobar degeneration and amyotrophic lateral sclerosis. Science. 2006;314(5796):130-133.

3. Arai T, et al. TDP-43 is a component of ubiquitinpositive tau-negative inclusions in frontotemporal lobar degeneration and amyotrophic lateral sclerosis. Biochem Biophys Res Commun. 2006;351(3):602-611.

4. Lagier-Tourenne C, Polymenidou M, Cleveland DW. TDP-43 and FUS/TLS: emerging roles in RNA processing and neurodegeneration. Hum Mol Genet. 2010;19(R1):R46-R64.

5. Mackenzie IR, Rademakers R, Neumann M. TDP-43 and FUS in amyotrophic lateral sclerosis and frontotemporal dementia. Lancet Neurol. 2010;9(10):995-1007.

6. Kwiatkowski TJ Jr, et al. Mutations in the FUS/TLS gene on chromosome 16 cause familial amyotrophic lateral sclerosis. Science. 2009;323(5918):1205-1208.

7. Vance C, et al. Mutations in FUS, an RNA processing protein, cause familial amyotrophic lateral sclerosis type 6. Science. 2009;323(5918):1208-1211.

8. Hewitt C, et al. Novel FUS/TLS mutations and pathology in familial and sporadic amyotrophic lateral sclerosis. Arch Neurol. 2010;67(4):455-461.

9. Dormann D, et al. ALS-associated fused in sarcoma (FUS) mutations disrupt Transportin-mediated nuclear import. EMBO J. 2010;29(16):2841-2857.

10. Kino Y, et al. Intracellular localization and splicing regulation of FUS/TLS are variably affected by amyotrophic lateral sclerosis-linked mutations. Nucleic Acids Res. 2011;39(7):2781-2798.

11. Deng HX, et al. FUS-immunoreactive inclusions are a common feature in sporadic and non-SOD1 familial amyotrophic lateral sclerosis. Ann Neurol. 2010;67(6):739-748.

12. Ling SC, et al. ALS-associated mutations in TDP43 increase its stability and promote TDP-43 complexes with FUS/TLS. Proc Natl Acad Sci U S A. 2010;107(30):13318-13323.

13. van Blitterswijk M, Landers JE. RNA processing pathways in amyotrophic lateral sclerosis. Neurogenetics. 2010;11(3):275-290.

14. Kim SH, Shanware NP, Bowler MJ, Tibbetts RS. Amyotrophic lateral sclerosis-associated proteins TDP-43 and FUS/TLS function in a common biochemical complex to co-regulate HDAC6 mRNA. J Biol Chem. 2010;285(44):34097-34105.

15. Lagier-Tourenne C, Cleveland DW. Rethinking ALS: the FUS about TDP-43. Cell. 2009;136(6):1001-1004.

16 . Sun $Z$, et al. Molecular determinants and genetic modifiers of aggregation and toxicity for the ALS disease protein FUS/TLS. PLoS Biol. 2011; 9(4):e1000614.

17. Stolow DT, Haynes SR. Cabeza, a Drosophila gene encoding a novel RNA binding protein, shares homology with EWS and TLS, two genes involved in human sarcoma formation. Nucleic Acids Res. 1995;23(5):835-843.

18. Elliott DA, Brand AH. The GAL4 system: a versatile system for the expression of genes. Methods Mol Biol. 2008;420:79-95.

19. Bischof J, Basler K. Recombinases and their use in gene activation, gene inactivation, and transgenesis. Methods Mol Biol. 2008;420:175-195.

20. Watson MR, Lagow RD, Xu K, Zhang B, Bonini NM. A drosophila model for amyotrophic lateral sclerosis reveals motor neuron damage by human SOD1. J Biol Chem. 2008;283(36):24972-24981.

21. Feiguin F, Godena VK, Romano G, D’Ambrogio A, Klima R, Baralle FE. Depletion of TDP-43 affects Drosophila motoneurons terminal synapsis and locomotive behavior. FEBS Lett. 2009;583(10):1586-1592.

22. Freibaum BD, Chitta RK, High AA, Taylor JP. Global analysis of TDP-43 interacting proteins reveals strong association with RNA splicing and translation machinery. J Proteome Res. 2010;9(2):1104-1120.

23. Buratti E, Baralle FE. The molecular links between TDP-43 dysfunction and neurodegeneration. Adv Genet. 2009;66:1-34.

24. Ju S, et al. A Yeast Model of FUS/TLS-Dependent Cytotoxicity. PLoS Biol. 2011;9(4):e1001052.

25. DeJesus-Hernandez M, et al. De novo truncating FUS gene mutation as a cause of sporadic amyotrophic lateral sclerosis. Hum Mutat. 2010;31(5):E1377-E1389.

26. Bosco DA, et al. Mutant FUS proteins that cause amyotrophic lateral sclerosis incorporate into stress granules. Hum Mol Genet. 2010;19(21):4160-4175.

27. Wegorzewska I, Bell S, Cairns NJ, Miller TM, Baloh RH. TDP-43 mutant transgenic mice develop features of ALS and frontotemporal lobar degeneration. Proc Natl Acad Sci U S A. 2009;106(44):18809-18814.

28. Kabashi E, et al. Gain and loss of function of ALS-related mutations of TARDBP (TDP-43) cause motor deficits in vivo. Hum Mol Genet. 2010;19(4):671-683.

29. Li Y, et al. A Drosophila model for TDP-43 proteinopathy. Proc Natl Acad Sci US A. 2010;107(7):3169-3174.

30. Wils H, et al. TDP-43 transgenic mice develop spastic paralysis and neuronal inclusions characteristic of ALS and frontotemporal lobar degeneration. Proc Natl Acad Sci U S A. 2010;107(8):3858-3863.

31. Hanson KA, Kim SH, Wassarman DA, Tibbetts RS. Ubiquilin modifies TDP-43 toxicity in a Drosophila model of amyotrophic lateral sclerosis (ALS). J Biol Chem. 2010;285(15):11068-11072.

32. Miguel L, Frébourg T, Campion D, Lecourtois M. Both cytoplasmic and nuclear accumulations of the protein are neurotoxic in Drosophila models of TDP-43 proteinopathies. Neurobiol Dis. 2011;41(2):398-406.

33. Voigt A, et al. TDP-43-mediated neuron loss in vivo requires RNA-binding activity. PLoS One. 2010;5(8):e12247.

34. Elden AC, et al. Ataxin-2 intermediate-length polyglutamine expansions are associated with increased risk for ALS. Nature. 2010;466(7310):1069-1075.

35. Igaz LM, et al. Dysregulation of the ALS-associated gene TDP-43 leads to neuronal death and degeneration in mice. J Clin Invest. 2011;121(2):726-738.

36. Rorth P. A modular misexpression screen in Drosophila detecting tissue-specific phenotypes. Proc Natl Acad Sci U S A. 1996;93(22):12418-12422.

37. Lin DM, Goodman CS. Ectopic and increased expression of Fasciclin II alters motoneuron growth 
cone guidance. Neuron. 1994;13(3):507-523.

38. Groth AC, Fish M, Nusse R, Calos MP. Construction of transgenic Drosophila by using the sitespecific integrase from phage phiC31. Genetics. 2004;166(4):1775-1782.

39. Freeman M. Reiterative use of the EGF receptor triggers differentiation of all cell types in the Drosophila eye. Cell. 1996;87(4):651-660.

40. Aberle H, Haghighi AP, Fetter RD, McCabe BD, Magalhaes TR, Goodman CS. wishful thinking encodes a BMP type II receptor that regulates synaptic growth in Drosophila. Neuron. 2002; 33(4):545-558

41. Pinnola A, Naumova N, Shah M, Tulin AV. Nucleosomal core histones mediate dynamic regulation of poly(ADP-ribose) polymerase 1 protein binding to chromatin and induction of its enzymatic activity. J Biol Chem. 2007;282(44):32511-32519.
42. Slawson JB, Kim EZ, Griffith LC. High-resolution video tracking of locomotion in adult Drosophila melanogaster. J Vis Exp. 2009;(24):1096.

43. Martinez VG, Javadi CS, Ngo E, Ngo L, Lagow RD, Zhang B. Age-related changes in climbing behavior and neural circuit physiology in Drosophila. Dev Neurobiol. 2007;67(6):778-791

44. Wu JS, Luo L. A protocol for dissecting Drosophila melanogaster brains for live imaging or immunostaining. Nat Protoc. 2006;1(4):2110-2115.

45. Brent JR, Werner KM, McCabe BD. Drosophila larval NMJ immunohistochemistry. J Vis Exp. 2009;(25):1108.

46. Brent JR, Werner KM, McCabe BD. Drosophila larval NMJ dissection. J Vis Exp. 2009;(24):1107.

47. Einhauer A, Jungbauer A. The FLAG peptide, a versatile fusion tag for the purification of recombinant proteins. J Biochem Biophys Methods. 2001;
49(1-3):455-465

48. Kuhn EJ, Viering MM, Rhodes KM, Geyer PK. A test of insulator interactions in Drosophila. EMBO J. 2003;22(10):2463-2471.

49. Bischof J, Maeda RK, Hediger M, Karch F, Basler K. An optimized transgenesis system for Drosophila using germ-line-specific phiC31 integrases. Proc Natl Acad SciU S A. 2007;104(9):3312-3317.

50. Pfeiffer BD, et al. Tools for neuroanatomy and neurogenetics in Drosophila. Proc Natl Acad Sci U S A. 2008;105(28):9715-9720.

51. Hartley JL, Temple GF, Brasch MA. DNA cloning using in vitro site-specific recombination. Genome Res. 2000;10(11):1788-1795.

52. Nagai T, Ibata K, Park ES, Kubota M, Mikoshiba K, Miyawaki A. A variant of yellow fluorescent protein with fast and efficient maturation for cell-biological applications. Nat Biotechnol. 2002;20(1):87-90. 